\title{
Copeptin and the risk of incident stroke, CHD and cardiovascular mortality in older men with and without diabetes: The British Regional Heart Study
}

\author{
S. Goya Wannamethee ${ }^{1} \cdot$ Paul Welsh $^{2} \cdot$ Lucy Lennon $^{1} \cdot$ Olia Papacosta $^{1}$. \\ Peter H. Whincup ${ }^{3}$ Naveed Sattar ${ }^{2}$
}

Received: 19 January 2016 / Accepted: 5 May 2016/Published online: 16 June 2016

(C) The Author(s) 2016. This article is published with open access at Springerlink.com

\begin{abstract}
Aims/hypothesis This study aimed to examine the association between copeptin (a surrogate marker of arginine vasopressin) and incident stroke, CHD and cardiovascular mortality in older men with and without diabetes.

Methods We conducted a prospective study of 3536 men aged 60-79 years who were followed for an average of 13 years. During this period, there were 437 major CHD events (fatal and non-fatal myocardial infarction [MI]), 323 stroke events (fatal and non-fatal) and 497 cardiovascular disease (CVD) deaths. Prevalent diabetes was defined on the basis of a history of doctor-diagnosed diabetes or fasting blood glucose $\geq 7.0 \mathrm{mmol}$ or $\mathrm{HbA}_{1 \mathrm{c}} \geq 6.5 \%(48 \mathrm{mmol} / \mathrm{mol})(n=428)$.

Results No association was seen between copeptin and incident stroke or CVD mortality in men without diabetes after adjustment for conventional cardiovascular risk factors, renal dysfunction, and insulin and $\mathrm{N}$-terminal pro B-type natriuretic peptide levels. In contrast, elevated copeptin levels were associated with an increased risk of stroke and CVD mortality in men with diabetes after these adjustments. Compared with those in the lowest tertile of copeptin, men in the top tertile had adjusted relative HRs for stroke and CVD
\end{abstract}

S. Goya Wannamethee

g.wannamethee@ucl.ac.uk

1 Department of Primary Care and Population Health, UCL Medical School, Royal Free Campus, Rowland Hill St, London NW3 2PF, UK

2 Institute of Cardiovascular and Medical Sciences, BHF Glasgow Cardiovascular Research Centre, University of Glasgow, Glasgow, UK

3 Population Health Research Institute, St George's, University of London, London, UK death of 2.34 (95\% CI 1.04, 5.27) and $2.21(1.12,4.36)$, respectively. The risk of stroke and CVD mortality remained increased after the exclusion of men with prevalent stroke or MI. Higher levels of copeptin were associated with increased risk of CHD in the diabetic and non-diabetic groups, but these associations were attenuated after exclusion of individuals with a previous stroke or MI.

Conclusions/interpretation Copeptin was independently associated with an increased risk of incident stroke and CVD mortality in men with diabetes, but not in men without diabetes. Targeting the arginine vasopressin system might have beneficial effects on CVD mortality and stroke risk in older men with diabetes.

Keywords Cardiovascular mortality · Copeptin · Coronary heart disease $\cdot$ Diabetes $\cdot$ Epidemiology $\cdot$ Stroke

$\begin{array}{ll}\text { Abbreviations } \\ \text { AVP } & \text { Arginine vasopressin } \\ \text { CRP } & \text { C-reactive protein } \\ \text { CVD } & \text { Cardiovascular disease } \\ \text { eGFR } & \text { Estimated GFR } \\ \text { MI } & \text { Myocardial infarction } \\ \text { NT-proBNP } & \text { N-terminal pro B-type natriuretic peptide } \\ \text { SGLT2 } & \text { Sodium-glucose transporter 2 }\end{array}$

\section{Introduction}

Cardiovascular disease (CVD) is the leading cause of death in patients with type 2 diabetes mellitus [1,2]. It is well established that type 2 diabetes is a major risk factor for CVD, including myocardial infarction (MI) and stroke [1-3]. Type 2 diabetes has been associated with a two- to 
sixfold increase in the risk of stroke compared with no diabetes [3]. The reduction of cardiovascular risk in individuals with type 2 diabetes is thus of paramount importance.

Arginine vasopressin (AVP), also known as antidiuretic hormone, is a nonapeptide that is produced in the hypothalamus. AVP is released from the neurohypophysis to promote renal water conservation, and plays important roles in the regulation of the cardiovascular system, water electrolyte balance and many functions of the central nervous system $[4,5]$. Vasopressin receptors are widely distributed throughout the brain [6]. They are present in neurons, astrocytes and their perivascular processes, blood vessel endothelial and smooth muscle cells, and the choroid plexus. These locations suggest that vasopressin may participate in regulating vascular resistance in the cerebral circulation and water homeostasis in the brain [6]. Human and animal studies have indicated that the AVP system may play a role in glucose homeostasis, insulin resistance, and lipid and fat metabolism [7-9], and vasopressin has been reported to be elevated in patients with type 2 diabetes [10]. However, plasma AVP is difficult to measure because of its instability and short half-life [11]. Plasma carboxy-terminal pro-vasopressin (copeptin), the C-terminal part of the AVP precursor peptide, is stable in serum and plasma, making it a more convenient biomarker. Indeed, copeptin has been established as a reliable marker of the circulating AVP concentration in routine clinical practice [11].

Copeptin is known to be raised in individuals with type 2 diabetes and has been associated with the development of diabetes $[12,13]$. Raised copeptin has also been associated with CVD mortality in patients with type 2 diabetes complicated by end-stage renal disease or acute MI, as well as in individuals with diabetes who are treated in primary care and those in the general population [14-18]. Stroke incidence rises steeply with age and the number of older people with type 2 diabetes is increasing. Attention has therefore turned to identifying new therapeutic approaches to preventing stroke in individuals with type 2 diabetes [19]. Copeptin has been shown to predict incident stroke in diabetic patients undergoing haemodialysis [15], but its ability to predict incident stroke events in individuals with and without diabetes among the older general population has not yet been assessed. To evaluate these issues, we examined the association between copeptin and incident stroke, as well as CHD events (fatal and non-fatal) and CVD mortality, in men with and without diabetes.

\section{Methods}

Participants The British Regional Heart Study is a prospective study of CVD involving 7735 men aged
40-59 years drawn from general practice in each of 24 British towns, with participants recruited between 1978 and 1980 [20]. The population is socioeconomically representative of British men, but is predominantly comprised of white Europeans ( $>99 \%)$. In 1998-2000, all of the surviving men, then aged 60-79 years (mean age 68.7 years), were invited for a 20th year follow-up examination. The men completed a questionnaire, which included questions on their medical history and lifestyle. They were also asked to fast for a minimum of $6 \mathrm{~h}$, during which time they were instructed to drink only water, and to attend for various measurements (see below) at a prespecified time between 08:00 and 18:00 hours. All men were asked to provide a blood sample, which was collected using the Sarstedt Monovette system (Sarstedt, Numbrecht, Germany). The samples were frozen and stored at $-20^{\circ} \mathrm{C}$ on the day of collection and transferred in batches for storage at $-70^{\circ} \mathrm{C}$ until analysis, which was carried out after no more than one freeze-thaw cycle. The men were asked whether a doctor had ever told them that they had angina or MI (heart attack, coronary thrombosis) or stroke, and to take any medication they were taking to the examination session. A total of 4252 men ( $77 \%$ of survivors) underwent examination and 4094 provided blood samples.

Cardiovascular risk factors Anthropometric measurements, including body weight, height and waist circumference, were taken. Participants were measured standing in light clothing without shoes. Height was measured using a Harpenden stadiometer (Holtain, Crymych, UK) to the last complete $0.1 \mathrm{~cm}$, and weight was measured using a Soehnle digital electronic scale (Soehnle, Murrhardt, Germany) to the last complete $0.1 \mathrm{~kg}$. BMI (weight $/ \mathrm{height}^{2}\left[\mathrm{~kg} / \mathrm{m}^{2}\right]$ ) was calculated for each participant. Waist circumference was measured in duplicate using an insertion tape (CMS, London, UK) at the midpoint between the iliac crest and the lower rib margins measured at the sides. Details of measurements and classification methods for smoking status, physical activity, social class and BP have been previously described [21, 22].

Moderate drinking was defined as 21-34 drinks/week (3-4 drinks/day), while heavy drinking was defined as those reporting drinking $\geq 35$ drinks/week ( $\geq 5$ drinks/day). Plasma glucose was measured with a glucose oxidase method using a Falcor 600 automated analyser (A. Menarini Diagnostics, Wokingham, UK) [23]. Serum insulin was measured using an ELISA assay (R\&D systems, Oxford UK) that did not cross-react with proinsulin [24]. Triacylglycerol, glucose and insulin concentrations were adjusted for the effects of fasting duration and time of day [22]. Insulin resistance was estimated according to HOMA [25]. The estimated (e)GFR (a measure of renal function) was calculated from serum creatinine using the modification of diet in renal disease equation developed by Levey et al [26]. Chronic kidney disease was defined as eGFR 
$<60 \mathrm{ml} \mathrm{min}^{-1} 1.73 \mathrm{~m}^{-2}$. Von Willebrand factor antigen was measured using an ELISA antigen (DAKO, High Wycombe, UK). C-reactive protein (CRP) was assayed by ultra-sensitive nephelometry (Dade Behring, Milton Keynes, UK).

Prevalent diabetes Men with a doctor's diagnosis of diabetes, those with fasting glucose of $\geq 7 \mathrm{mmol} / \mathrm{l}$ (WHO criteria) or those with $\mathrm{HbA}_{1 \mathrm{c}} \geq 6.5 \%$ (48 $\left.\mathrm{mmol} / \mathrm{mol}\right)$ were considered to have prevalent diabetes [27].

Copeptin and N-terminal pro B-type natriuretic peptide (NT-proBNP) Serum levels of copeptin and NT-proBNP were measured using manufacturers' calibrators and controls in accordance with their instructions. NT-proBNP was determined using the Elecsys 2010 electrochemiluminescence method (Roche Diagnostics, Burgess Hill, UK) [28]. The lower limit of sensitivity was $5 \mathrm{ng} / \mathrm{l}$. Low control CV was $6.7 \%$ and high control CV was $4.9 \%$. Copeptin was measured using an ultra-sensitive method on a BRAHMS Kryptor Compact Plus (BRAHMS, Bottisham, UK). The lower limit of sensitivity was $0.9 \mathrm{pmol} / \mathrm{l}$. Low control CV was $4.7 \%$ and high control CV was $4.6 \%$. We examined copeptin levels by the time of examination. Although copeptin levels were highest at 13:00 hours (geometric mean $4.95 \mathrm{pmol} / \mathrm{l}$, interquartile range [IQR] $2.76-8.11 \mathrm{pmol} / \mathrm{l}$ ), there was no consistent significant diurnal variation throughout the day. For example, the mean copeptin level between 09:00 and 10:00 hours (3.97 pmol/1 [IQR 2.50-6.58 pmol/1]) was similar to that between 12:00 and 13:00 hours (3.94 pmol/1 [IQR 2.49-6.49 pmol/1]) and between 17:00 and 18:00 hours (3.94 pmol/1 [IQR 2.61-6.32 pmol/1]).

Follow-up All of the men were followed for cardiovascular morbidity from the initial examination (1978-1980), and follow-up was achieved for $99 \%$ of the cohort [29]. In the present analyses, all-cause mortality and morbidity events were based on follow-up from re-screening in 1998-2000 at a mean age of 60-79 years to July 2012, a mean follow-up period of 13 years (range 12-14 years). Information on death was collected through National Health Service registers, in which the participants were flagged for follow-up [30]. During the follow-up period, changing diagnostic criteria were taken into account by using both ICD-9 and ICD-10 codes. For the present paper, CHD deaths were defined as ICD-9 codes $410-414$ or ICD-10 codes I20-I25. Stroke deaths were defined as ICD-9 codes $430-438$ or ICD-10 codes I60-I69. Non-fatal stroke events were defined as those that resulted in a neurological deficit that was present for more than $24 \mathrm{~h}$. Evidence regarding non-fatal stroke and MI events was obtained by ongoing reports from general practitioners, by biennial reviews of patients' practice records (including hospital and clinic correspondence) through to the end of the study period and from repeated questionnaires sent to surviving participants after the initial examination. Pre-existing CVD was defined as a history of a doctor's diagnosis of MI or stroke. Patient recall of a doctor's diagnosis of CHD has been shown to be a valid measure of recording disease in this study population [31]; the $\mathrm{K}$ statistic for comparing record reviews with patients' recall of CHD was $0.82[31]$.

Statistical methods The distribution of copeptin levels was skewed and natural log transformation was therefore used. Cox's proportional hazards model was used to assess multivariate-adjusted HRs (relative risk) in a comparison of three copeptin groups $(<3.35,3.35-6.95$ and $\geq 6.96 \mathrm{pmol} / \mathrm{l})$, as well as in a 1 unit increase in $\log _{e}$ copeptin. As men with diabetes showed significantly higher levels of copeptin than men without diabetes (mean $6.96 \mathrm{pmol} / 1$ [IQR $2.90-8.63 \mathrm{pmol} / 1]$ vs $3.34 \mathrm{pmol} / 1$ [IQR $2.40-5.88 \mathrm{pmol} / 1]$, respectively), the cut-offs for the three groups were based on the tertile distribution in men with diabetes to achieve sufficient numbers in each group and then applied to those without diabetes. Thus, the number of men in each of the three groups was not equally distributed in those without diabetes.

In multivariate analyses, smoking (never, long-term ex-smokers [ $>15$ years], recent ex-smokers $[<15$ years] and current smokers), social class (manual vs non-manual work), physical activity (four groups), heavy drinking (yes/no), prevalent MI and prevalent stroke were fitted as categorical variables; CRP, eGFR, BMI, systolic BP, HDL-cholesterol, HOMA-IR, blood glucose and NT-proBNP were fitted as continuous variables. Adjustment for diurnal variation was carried out by fitting the time of examination as three groups: morning (9:00-13:00 hours), lunchtime (13:00 hours) and afternoon (14:00-18:00 hours). A test for interaction was carried out by fitting the interaction term ' $\log _{e}$ copeptin $\times$ prevalent diabetes (yes/no)' into the model. A sensitivity analysis was performed excluding men with prevalent MI or stroke. $p<0.05$ was used to indicate statistical significance.

Study sample Copeptin measurements were available for 3663 men. Because heart failure is known to raise AVP (copeptin) and copeptin may be a marker of the severity of heart failure [32], we excluded 127 men with a history of heart failure, leaving 3536 men for analysis.

\section{Results}

During the mean follow-up period of 13 years, the 3536 men without heart failure experienced 437 major CHD events (11.3/1000 person-years) and 323 major stroke events (8.6/1000 person-years), with 497 deaths from cardiovascular causes. 


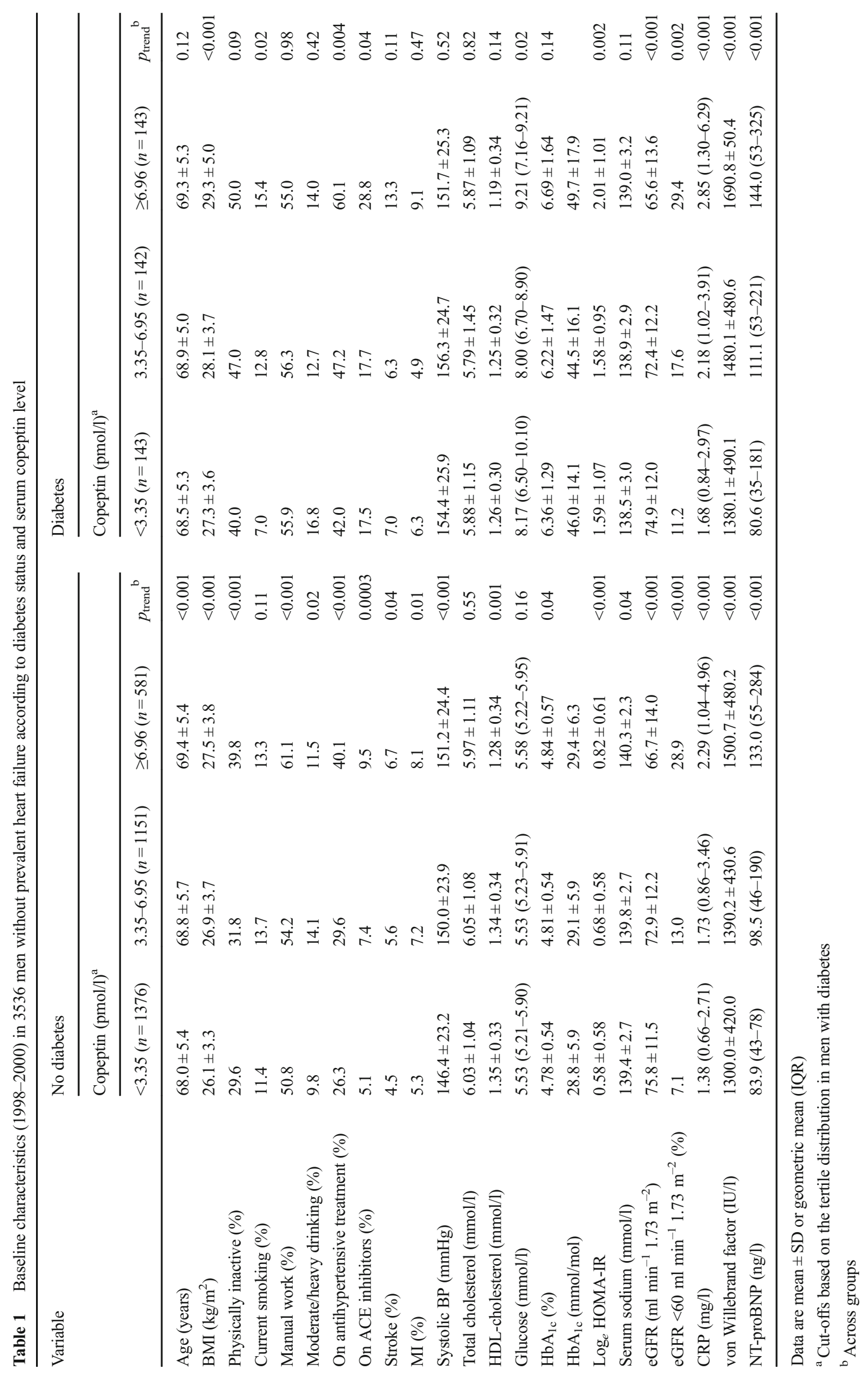


Table 1 shows baseline characteristics by the three copeptin groups in men with and without diabetes. Copeptin was strongly related to BMI, renal function, HOMA-IR and NT-proBNP in both the diabetic and non-diabetic groups. The prevalence of stroke and MI was highest in those with elevated copeptin.

\section{Copeptin and incident CVD in men without diabetes} Elevated copeptin was associated with a significantly increased risk of CHD events after adjustment for age (Table 2). Further adjustment for CVD risk factors including smoking, physical activity, social class, BMI, HDL-C, systolic $\mathrm{BP}$, antihypertensive treatment, eGFR, prevalent MI, prevalent stroke, CRP, HOMA-IR and blood glucose (Model 2 ) attenuated the association, although it remained statistically significant. However, the association was markedly reduced after the exclusion of men with prevalent CVD (MI or stroke). There was no significant association between copeptin and incident stroke or CVD mortality after adjustment for these CVD risk factors in Model 2. Additional adjustment for diurnal variations made little difference to the findings.
Copeptin and incident CVD in men with diabetes In contrast to men without diabetes, copeptin was significantly associated with incident stroke and CVD mortality among men with diabetes, even after adjustment for cardiovascular risk factors and upon the exclusion of men with prevalent CVD (Table 3). Additional adjustment for the use of ACE inhibitors made little difference to these findings. A formal test for interaction confirmed a significant difference in the relationship between copeptin and risk of stroke and CVD between men with and without diabetes after the exclusion of men with MI or stroke $(p=0.01$ and $p=0.02$, respectively).

No association was seen between copeptin and the risk of major CHD events (fatal or non-fatal) in men with diabetes after adjustment for established cardiovascular risk factors (Table 3). In contrast to the significant interaction seen for stroke and CVD mortality, there was no significant interaction for CHD events between men with and without diabetes $(p=0.81)$. Additional adjustment for diurnal variation made no material difference to the findings. When examined in relation to non-fatal $(n=19)$ and fatal $(n=48)$ CHD events, a
Table 2 Incidence rates $/ 1000$ person-years and adjusted HRs (95\% CIs) for CVD endpoints by copeptin levels in 3108 men with no prevalent diabetes and no heart failure

\begin{tabular}{|c|c|c|c|c|c|}
\hline \multirow[t]{2}{*}{ Incidence } & \multicolumn{3}{|c|}{ Copeptin $(\mathrm{pmol} / \mathrm{l})^{\mathrm{a}}$} & \multirow{2}{*}{$\begin{array}{l}1 \text { unit increase } \\
\text { in } \log _{e} \text { copeptin }\end{array}$} & \multirow[t]{2}{*}{$p$ trend } \\
\hline & $\begin{array}{l}<3.35 \\
(n=1376)\end{array}$ & $\begin{array}{l}3.35-6.95 \\
(n=1151)\end{array}$ & $\begin{array}{l}\geq 6.96 \\
(n=581)\end{array}$ & & \\
\hline \multicolumn{6}{|l|}{$\mathrm{CHD}(n=370)$} \\
\hline $\begin{array}{l}\text { Rate/1000 } \\
\text { person-years }(n)\end{array}$ & $8.5(134)$ & $11.4(146)$ & $15.2(90)$ & & \\
\hline Age-adjusted HR & 1.00 & $1.27(1.01,1.61)$ & $1.68(1.28,2.19)$ & $1.23(1.00,1.40)$ & 0.002 \\
\hline Model 1 & 1.00 & $1.24(0.97,1.57)$ & $1.45(1.10,1.92)$ & $1.15(1.01,1.30)$ & 0.04 \\
\hline Model 2 & 1.00 & $1.21(0.95,1.55)$ & $1.36(1.01,1.83)$ & $1.12(0.98,1.27)$ & 0.10 \\
\hline Model 2a & 1.00 & $1.24(0.94,1.62)$ & $1.22(0.87,1.71)$ & $1.04(0.91,1.19)$ & 0.54 \\
\hline \multicolumn{6}{|l|}{ Stroke $(n=272)$} \\
\hline $\begin{array}{l}\text { Rate/1000 } \\
\text { person-years }(n)\end{array}$ & $7.2(111)$ & $8.2(101)$ & $10.5(60)$ & & \\
\hline Age-adjusted HR & 1.00 & $1.07(0.81,1.40)$ & $1.37(1.00,1.84)$ & $1.12(0.97,1.29)$ & 0.13 \\
\hline Model 1 & 1.00 & $1.00(0.76,1.32)$ & $1.16(0.84,1.62)$ & $1.03(0.90,1.17)$ & 0.65 \\
\hline Model 2 & 1.00 & $0.99(0.75,1.31)$ & $1.13(0.80,1.59)$ & $1.03(0.90,1.17)$ & 0.71 \\
\hline Model 2a & 1.00 & $1.12(0.82,1.53)$ & $1.26(0.79,1.71)$ & $1.05(0.90,1.24)$ & 0.52 \\
\hline \multicolumn{6}{|l|}{ CVD death $(n=419)$} \\
\hline $\begin{array}{l}\text { Rate/1000 } \\
\text { person-years }(n)\end{array}$ & $9.2(144)$ & $13.2(168)$ & $18.1(107)$ & & \\
\hline Age-adjusted HR & 1.00 & $1.31(1.05,1.64)$ & $1.82(1.41,2.33)$ & $1.29(1.14,1.46)$ & 0.005 \\
\hline Model 1 & 1.00 & $1.18(0.93,1.48)$ & $1.43(1.10,1.86)$ & $1.13(1.00,1.28)$ & 0.05 \\
\hline Model 2 & 1.00 & $1.15(0.91,1.45)$ & $1.28(0.97,1.69)$ & $1.08(0.95,1.22)$ & 0.22 \\
\hline Model 2a & 1.00 & $1.19(0.96,1.60)$ & $1.02(0.73,1.43)$ & $0.97(0.86,1.11)$ & 0.62 \\
\hline
\end{tabular}

${ }^{\text {a }}$ Cut-offs based on the tertile distribution in men with diabetes

Model 1: adjusted for age, smoking, physical activity, heavy drinking, social class, BMI, HDL-C, systolic BP, antihypertensive treatment, prevalent $\mathrm{MI}$ and prevalent stroke

Model 2: Model 1 + CRP + HOMA-IR + blood glucose + eGFR

Model 2a: adjusted for Model 1 and excluding men with prevalent MI/stroke $(n=353 ; 11.4 \%)$ 
Table 3 Incidence rates $/ 1000$ person-years and adjusted HRs (95\% CIs) for CVD endpoints by copeptin levels in 428 men with prevalent diabetes and no heart failure

\begin{tabular}{|c|c|c|c|c|c|}
\hline \multirow[t]{2}{*}{ Incidence } & \multicolumn{3}{|c|}{ Copeptin $(\mathrm{pmol} / \mathrm{l})^{\mathrm{a}}$} & \multirow{2}{*}{$\begin{array}{l}1 \text { unit increase } \\
\text { in } \log _{e} \text { copeptin }\end{array}$} & \multirow[t]{2}{*}{$p$ trend } \\
\hline & $\begin{array}{l}<3.35 \\
(n=143)\end{array}$ & $\begin{array}{l}3.35-6.95 \\
(n=142)\end{array}$ & $\begin{array}{l}\geq 6.96 \\
(n=143)\end{array}$ & & \\
\hline \multicolumn{6}{|l|}{$\mathrm{CHD}(n=67)$} \\
\hline $\begin{array}{l}\text { Rate/1000 } \\
\text { person-years }(n)\end{array}$ & $12.5(20)$ & $13.6(19)$ & $22.5(28)$ & & \\
\hline Age-adjusted HR & 1.00 & $1.11(0.59,2.05)$ & $1.85(1.04,3.29)$ & $1.17(0.86,1.59)$ & 0.33 \\
\hline Model 1 & 1.00 & $0.95(0.49,1.82)$ & $1.59(0.83,3.04)$ & $1.02(0.77,1.36)$ & 0.88 \\
\hline Model 2 & 1.00 & $0.88(0.45,1.73)$ & $1.21(0.60,2.42)$ & $0.94(0.72,1.22)$ & 0.63 \\
\hline Model 3 & 1.00 & $0.86(0.43,1.68)$ & $1.18(0.59,2.35)$ & $0.94(0.73,1.20)$ & 0.59 \\
\hline Model 3a & 1.00 & $0.76(0.36,1.60)$ & $0.99(0.47,2.09)$ & $1.00(0.75,1.33)$ & 0.99 \\
\hline \multicolumn{6}{|l|}{ Stroke $(n=51)$} \\
\hline $\begin{array}{l}\text { Rate/1000 } \\
\text { person-years }(n)\end{array}$ & $7.7(12)$ & $11.2(15)$ & $20.5(24)$ & & \\
\hline Age-adjusted HR & 1.00 & $1.49(0.70,3.18)$ & $2.69(1.34,5.40)$ & $1.87(1.29,2.71)$ & 0.001 \\
\hline Model 1 & 1.00 & $1.96(0.88,4.36)$ & $2.34(1.11,4.91)$ & $1.62(1.11,2.37)$ & 0.01 \\
\hline Model 2 & 1.00 & $2.11(0.93,4.76)$ & $2.22(1.01,4.89)$ & $1.63(1.07,2.48)$ & 0.02 \\
\hline Model 3 & 1.00 & $2.16(0.93,4.98)$ & $2.34(1.04,5.27)$ & $1.65(1.06,2.55)$ & 0.03 \\
\hline Model 3a & 1.00 & $2.03(0.71,5.81)$ & $2.97(1.09,8.12)$ & $1.73(0.98,3.03)$ & 0.05 \\
\hline \multicolumn{6}{|l|}{ CVD death $(n=78)$} \\
\hline $\begin{array}{l}\text { Rate/1000 } \\
\text { person-years }(n)\end{array}$ & $10.0(16)$ & $17.8(25)$ & $29.7(37)$ & & \\
\hline Age-adjusted HR & 1.00 & $1.77(0.94,3.31)$ & $2.78(1.54,4.99)$ & $1.79(1.33,2.39)$ & $<0.0001$ \\
\hline Model 1 & 1.00 & $1.67(0.88,3.18)$ & $2.99(1.57,5.71)$ & $1.62(1.18,2.23)$ & 0.003 \\
\hline Model 2 & 1.00 & $1.65(0.86,3.16)$ & $2.49(1.27,4.90)$ & $1.47(1.06,2.04)$ & 0.02 \\
\hline Model 3 & 1.00 & $1.55(0.80,3.03)$ & $2.21(1.12,4.36)$ & $1.34(0.98,1.84)$ & 0.06 \\
\hline Model 3a & 1.00 & $1.76(0.80,3.91)$ & $3.03(1.39,6.62)$ & $1.92(1.23,2.98)$ & 0.004 \\
\hline
\end{tabular}

${ }^{\text {a }}$ Cut-offs based on the tertile distribution in men with diabetes

Model 1: adjusted for age, smoking, heavy drinking, physical activity, social class, BMI, HDL-C, systolic BP, antihypertensive treatment, prevalent $\mathrm{MI}$ and prevalent stroke

Model 2: Model 1 + CRP + HOMA-IR + blood glucose + eGFR

Model 3: Model 2+ NT-proBNP

Model 3a: Model 3, excluding men with prevalent MI/stroke $(n=61 ; 14.3 \%)$ non-significant inverse association between copeptin levels and non-fatal events was seen, and a positive association was seen with fatal events after adjustment for factors in Model 3, although the numbers were small and the increased risk was not significant (adjusted HR 1.68 [95\% CI 0.72, 3.92]; top third vs lowest third). Although CHD deaths accounted for more than $50 \%$ of all CVD deaths $(n=48 / 78)$, the increased risk of CVD mortality was largely due to stroke or other CVD deaths (adjusted HR 4.64 [95\% CI 1.27, 16.74]).

\section{Discussion}

In this study of older men, copeptin was significantly associated with incident stroke and CVD mortality in men with diabetes, after adjustment for established CVD risk factors and following the exclusion of men with MI or stroke, and in keeping with the markedly elevated copeptin levels in this patient group. In contrast, we did not find any significant association between copeptin and incident stroke or CVD mortality in men without diabetes after adjustment for established CVD risk factors. Copeptin was positively associated with incident CHD in men both with and without diabetes, but these associations were to a large extent associated with pre-existing CVD as the exclusion of individuals with MI/stroke attenuated the association in both groups. Our findings confirm previous studies reporting an association between copeptin and CVD in diabetes [14-18], and extend the findings to stroke incidence in individuals both with and without diabetes in the general population. We were able to take into account a wide range of CVD risk markers, including insulin resistance, inflammation, renal function and NT-proBNP. 
Diabetes status, copeptin and CVD Our findings extend those of a recent report in which copeptin predicted CVD endpoints in individuals with diabetes only [18]. Vasopressin levels are increased in patients with diabetes, although the cause of this is still unclear [10]. Although copeptin has previously been associated with CVD or stroke in specific subgroups of patients with diabetes and MI [16] or on haemodialysis [15], we are not aware of any other studies that have examined the association between copeptin and stroke in the general population of older people with diabetes. In this study, we observed a strong association between copeptin and stroke events, but not CHD events, after taking into account a wide range of vascular risk factors and upon exclusion of those with MI or stroke. We also observed a non-significant positive association between copeptin and CHD in the diabetes group. Although the lack of significance in the diabetes group might be due to small numbers, the positive association with CHD was markedly attenuated upon exclusion of those with MI or stroke, and a similar pattern of attenuation was also seen in participants without diabetes. Thus the increased risk of CHD associated with copeptin seen in both the diabetic and non-diabetic groups appears to be largely due to pre-existing CVD. This is consistent with previous reports that have shown copeptin to predict CVD specifically in those with coronary artery disease [33, 34].

Similar findings have also been reported in the German Diabetes and Dialysis Study, in which copeptin predicted stroke but not MI in diabetes patients undergoing haemodialysis [15]. In the Malmo Diet and Cancer Study [18] a positive association was seen with CHD in the diabetes group, which included individuals who developed diabetes during follow-up as well as prevalent diabetes cases. We did not include incident diabetes in our population of participants with diabetes. However, when the Malmo Diet and Cancer Study restricted its analysis to prevalent diabetes only, there was no association between copeptin and CHD events, similar to our findings.

Copeptin and risk of major stroke events Brain injury, including ischaemia, causes the release of stress hormones such as AVP [35]. It is increasingly being recognised that copeptin predicts adverse outcomes in patients with stroke [36]. However, reports on the association between copeptin and incident stroke in the general population are lacking. Our findings show that copeptin is associated with the development of stroke, even in diabetes patients without prevalent stroke. Whether the relationship between copeptin and stroke risk in diabetes is causal is uncertain, but it was not explained by conventional risk factors for stroke, insulin resistance or NT-proBNP. Renal dysfunction has been associated with an increased risk of stroke [37] and AVP is known to be associated with declining kidney function in patients with diabetes [38]. However, the association between copeptin and stroke was seen after adjustment for eGFR and even after the exclusion of men with chronic kidney disease. Population-based studies have shown copeptin to be strongly associated with microalbuminuria [39], suggesting a role for the AVP system in the development of albuminuria, which has been linked to incident stroke in diabetes as well as in the general population $[39,40]$. This may be a consequence of the antidiuretic effect of vasopressin, which leads to increased urinary albumin excretion, an effect mediated by vasopressin 2 receptors $[38,41]$. Thus, the association between copeptin, which is a marker of endogenous vasopressin secretion, and incident stroke may be linked to increased albuminuria.

Our finding of an association between copeptin and CVD mortality and stroke events in individuals with diabetes, but not in those without diabetes, is of potential relevance to the recent findings of the EMPA-REG OUTCOME trial, particularly given prior associations of elevated copeptin with renal and cardiac pathology, heart failure and CVD mortality. EMPA-REG OUTCOME, which investigated the effects of the sodium-glucose transporter 2 (SGLT2) inhibitor empagliflozin on cardiovascular morbidity and mortality in patients with type 2 diabetes at high cardiovascular risk, demonstrated a substantial reduction in CVD death and heart failure hospitalisations with empagliflozin but no effect on non-fatal CVD events [42]. One may speculate that many diabetes patients with CVD have subclinical fluid overload, making them exquisitely sensitive to fluid balance changes. Notably, SGLT2 inhibitors promote glycosuria by lowering the renal glucose reabsorption threshold, whereas AVP promotes water reabsorption through stimulation of $V_{2}$ receptors, and increased levels of AVP appear to limit glucose-induced water loss in patients with diabetes [43]. These findings suggest that SGLT2 inhibitors should lessen copeptin levels in line with improved fluid balance and cardiac and renal function in diabetes patients with CVD, a hypothesis that should be directly testable in the near future.

Strengths and limitations The strengths and limitations of this study require consideration. This is the first study to examine the association between copeptin and incident stroke in a general population of older men with and without diabetes, who constitute a high-risk group for CVD. The population of the British Regional Heart Study, though moderate in size, is socially representative of the UK and follow-up rates are exceptionally high. Ascertainment of CHD death and MI in this study was based on standard methods, and both CHD mortality and MI incidence rates correspond closely with national data. We were able to take into account a wide range of cardiovascular risk factors, including markers of inflammation and cardiac markers. However, our study was based on an older, predominantly white European, male population, so the results cannot be generalised directly to women, younger men or other ethnic 
groups; the results therefore need to be substantiated in other studies and in different population groups. Furthermore, our findings are based on a single measurement at the baseline re-examination rather than on repeated assessments, which may have led to an underestimation of the true strength of associations. In addition, we did not have information on microalbuminuria. Although we did not have information on the type of stroke for all men, $85 \%$ of stroke cases in Great Britain are caused by cerebral ischaemia [44].

Conclusion In this study, we found a significant association between copeptin and subsequent stroke risk and CVD mortality in men with diabetes, but not in men without diabetes, with those with diabetes also having substantially elevated copeptin levels. Future studies are required to explore whether elevated copeptin, as a sensitive marker for AVP secretion, is just a risk marker or a potentially modifiable risk factor for CVD mortality and stroke in diabetes. The latter would suggest that suppressing vasopressin with vasopressin antagonists may have a potential role in reducing stroke and CVD mortality among individuals with diabetes.

Funding The British Regional Heart Study is a British Heart Foundation (BHF) research group and receives support from a BHF programme grant (RG/13/16/30528). Dr Paul Welsh is supported by BHF fellowship FS/12/62/29889. Copeptin measurements were funded by the School for Primary Care Research of the National Institute for Health Research.

Duality of interest statement The authors declare that there is no duality of interest associated with this manuscript.

Contribution statement SGW initiated the concept and design of the paper, analysed the data and drafted the manuscript. PW, PHW and NS contributed to the interpretation of data. OP contributed to the analysis of the paper. PW, LL and PHW contributed to data acquisition. All authors revised the manuscript critically for important intellectual content and approved the final version of the manuscript. SGW is the guarantor for the manuscript and has full access to all the data in the study, and takes responsibility for the integrity of the data and the accuracy of the data analysis.

Open Access This article is distributed under the terms of the Creative Commons Attribution 4.0 International License (http:// creativecommons.org/licenses/by/4.0/), which permits unrestricted use, distribution, and reproduction in any medium, provided you give appropriate credit to the original author(s) and the source, provide a link to the Creative Commons license, and indicate if changes were made.

\section{References}

1. Taylor KS, Heneghan CJ, Farmer AJ et al (2013) All-cause and cardiovascular mortality in middle-aged people with type 2 diabetes compared with people without diabetes in a large U.K. primary care database. Diabetes Care 36:2366-2371

2. Rydén L, Grant PJ, Anker SD et al (2013) ESC Guidelines on diabetes, pre-diabetes, and cardiovascular diseases developed in collaboration with the EASD: the Task Force on diabetes, pre-diabetes, and cardiovascular diseases of the European Society of Cardiology (ESC) and developed in collaboration with the European Association for the Study of Diabetes (EASD). Eur Heart J 34:3035-3087

3. Luitse MJ, Biessels GJ, Rutten GE et al (2012) Diabetes, hyperglycaemia and acute ischaemic stroke. Lancet Neurol 11: 261-271

4. Faraco G, Wijasa TS, Park L et al (2014) Water deprivation induces neurovascular and cognitive dysfunction through vasopressininduced oxidative stress. J Cereb Blood Flow Metab 34:852-860

5. Szinnai G, Morgenthaler NG, Berneis K et al (2007) Changes in plasma copeptin, the c-terminal portion of arginine vasopressin during water deprivation and excess in healthy subjects. J Clin Endocrinol Metab 92:3973-3978

6. Barrett LK, Singer M, Clapp LH (2007) Vasopressin mechanisms of action on the vasculature in health and in septic shock. Crit Care Med 35:33-40

7. Oshikawa S, Tanoue A, Koshimizu TA et al (2004) Vasopressin stimulates insulin release from islet cells through $\mathrm{V} 1 \mathrm{~b}$ receptors: a combined pharmacological/knockout approach. Mol Pharmacol 65: 623-629

8. Spruce BA, McCulloch AJ, Burd J et al (1985) The effect of vasopressin infusion on glucose metabolism in man. Clin Endocrinol (Oxf) 22:463-468

9. Hiroyama M, Fujiwara Y, Nakamura K et al (2009) Altered lipid metabolism in vasopressin V1B receptor-deficient mice. Eur J Pharmacol 602:455-461

10. Bankir L, Bardoux P, Ahloulay M (2001) Vasopressin and diabetes mellitus. Nephron 87:8-18

11. Morgenthaler NG, Struck J, Alonso C et al (2006) Assay for the measurement of copeptin, a stable peptide derived from the precursor of vasopressin. Clin Chem 52:112-119

12. Enhörning S, Bankir L, Bouby N et al (2013) Copeptin, a marker of vasopressin, in abdominal obesity, diabetes and microalbuminuria: the prospective Malmö Diet and Cancer Study cardiovascular cohort. Int J Obes (Lond) 37:598-603

13. Wannamethee SG, Welsh P, Papacosta O et al (2015) Copeptin, insulin resistance and risk of incident diabetes in older men. J Clin Endocrinol Metab 100:3332-3339

14. Maier C, Clodi M, Neuhold S et al (2009) Endothelial markers may link kidney function to cardiovascular events in type 2 diabetes. Diabetes Care 32:1890-1895

15. Fenske W, Wanner C, Allolio B et al (2011) Copeptin levels associate with cardiovascular events in patients with ESRD and type 2 diabetes mellitus. J Am Soc Nephrol 22:782-790

16. Mellbin LG, Rydén L, Brismar K et al (2010) Copeptin, IGFBP-1, and cardiovascular prognosis in patients with type 2 diabetes and acute myocardial infarction: a report from the DIGAMI 2 trial. Diabetes Care 33:1604-1606

17. Riphagen IJ, Boertien WE, Alkhalaf A et al (2013) Copeptin, a surrogate marker for arginine vasopressin, is associated with cardiovascular and all-cause mortality in patients with type 2 diabetes (ZODIAC-31). Diabetes Care 36:3201-3207

18. Enhörning S, Hedblad B, Nilsson PM et al (2015) Copeptin is an independent predictor of diabetic heart disease and death. Am Heart J 169:549-556

19. Sander D, Kearney MT (2009) Reducing the risk of stroke in type 2 diabetes: pathophysiological and therapeutic perspectives. J Neurol 256:1603-1609

20. Shaper AG, Pocock SJ, Walker M et al (1981) British Regional Heart Study: cardiovascular risk factors in middle-aged men in 24 towns. BMJ 283:179-186

21. Wannamethee SG, Lowe GD, Whincup PH et al (2002) Physical activity and hemostatic and inflammatory variables in elderly men. Circulation 105:1785-1790 
22. Emberson J, Whincup PH, Walker M et al (2002) Biochemical measures in a population based study: the effect of fasting duration and time of day. Ann Clin Biochem 39:493-501

23. Trinder P (1969) Determination of blood glucose in blood using glucose oxidase with an alternative oxygen acceptor. Ann Clin Biochem 6:24-27

24. Andersen L, Dinesen B, Jorgensen PN et al (1993) Enzyme immunoassay for intact human insulin in serum or plasma. Clin Chem 39:578-582

25. Matthews DR, Hosker JP, Rudenski AS et al (1985) Homeostasis model assessment: insulin resistance and beta-cell function from fasting plasma glucose and insulin concentrations in man. Diabetologia 28:412-419

26. Levey AS, Bosch JP, Lewis JB et al (1999) A more accurate method to estimate glomerular filtration rate from serum creatinine: a new prediction equation. Modification of Diet in Renal Disease Study Group. Ann Intern Med 130:461-470

27. International Expert Committee (2009) International Expert Committee report on the role of the A1c assay in the diagnosis of diabetes. Diabetes Care 32:1327-1334

28. Wannamethee SG, Welsh P, Whincup P et al (2014) N-terminal pro brain natriuretic peptide but not copeptin improves prediction of heart failure over other routine clinical risk parameters in older men with and without cardiovascular disease: population-based study. Eur J Heart Fail 16:25-32

29. Lennon LT, Ramsay SE, Papacosta O et al (2015) Cohort profile update: The British Regional Heart Study 1978-2014: 35 years follow-up of cardiovascular disease and ageing. Int J Epidemiol $44: 826-826 \mathrm{~g}$

30. Walker M, Shaper AG, Lennon L et al (2000) Twenty year follow-up of a cohort based in general practices in 24 British towns. J Public Health Med 22:479-485

31. Walker M, Whincup PH, Shaper AG et al (1998) Validation of patient recall of doctor diagnosed heart attack and stroke: a postal questionnaire and record review comparison. Am J Epidemiol 148: 355-361

32. Neuhold S, Huelsmann M, Strunk G et al (2008) Comparison of copeptin, B-type natriuretic peptide, and amino-terminal pro-B-type natriuretic peptide in patients with chronic heart failure: prediction of death at different stages of the disease. J Am Coll Cardiol 52:266-272
33. von Haehling S, Papassotiriou J, Morgenthaler NG et al (2012) Copeptin as a prognostic factor for major adverse cardiovascular events in patients with coronary artery disease. Int J Cardiol 162: 27-32

34. Potocki M, Reichlin T, Thalmann S et al (2012) Diagnostic and prognostic impact of copeptin and high-sensitivity cardiac troponin $\mathrm{T}$ in patients with pre-existing coronary artery disease and suspected acute myocardial infarction. Heart 98:558-565

35. Vakili A, Kataoka H, Plesnila N (2005) Role of arginine vasopressin $\mathrm{V} 1$ and $\mathrm{V} 2$ receptors for brain damage after transient focal cerebral ischaemia. J Cereb Blood Flow Metab 25: 1012-1019

36. Choi KS, Kim HJ, Chun HJ et al (2015) Prognostic role of copeptin after stroke: a systematic review and meta-analysis of observational studies. Sci Rep 5:11665

37. Lee M, Saver JL, Chang KH et al (2010) Low glomerular filtration rate and risk of stroke: meta-analysis. BMJ 341:c4249

38. Boertien WE, Riphagen IJ, Drion I et al (2013) Copeptin, a surrogate marker for arginine vasopressin, is associated with declining glomerular filtration in patients with diabetes mellitus (ZODIAC-33). Diabetologia 56:1680-1688

39. Meijer E, Bakker SJ, Halbesma $\mathrm{N}$ et al (2010) Copeptin, a surrogate marker of vasopressin, is associated with microalbuminuria in a large population cohort. Kidney Int 77:29-36

40. Yang X, Ko GT, So WY et al (2008) Additive interaction of hyperglycemia and albuminuria on risk of ischemic stroke in type 2 diabetes: Hong Kong Diabetes Registry. Diabetes Care 31: 2294-2300

41. Lee M, Saver JL, Chang KH et al (2010) Impact of microalbuminuria on incident stroke: a meta-analysis. Stroke 41: 2625-2631

42. Zinman B, Wanner C, Lachin JM et al (2015) Empagliflozin, cardiovascular outcomes, and mortality in type 2 diabetes. N Engl J Med 373(22):2117-2128

43. Bankir L, Fernandes S, Bardoux P et al (2005) Vasopressin-V2 receptor stimulation reduces sodium excretion in healthy humans. J Am Soc Nephrol 16:1920-1928

44. Sandercock PAG, Warlow CP, Jones LN et al (1989) Predisposing factors for cerebral infarction: the Oxfordshire Community Stroke Project. BMJ 298:75-80 